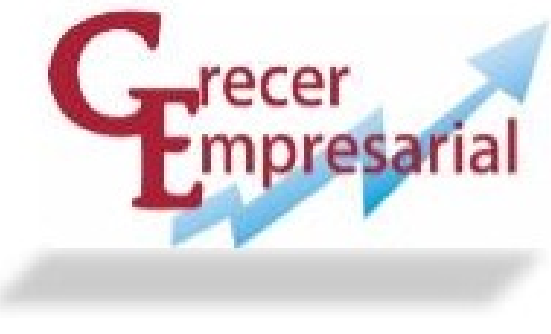

\title{
ANÁLISIS EMPÍRICO DE LAS CAUSAS DE LA INFLACIÓN EN COLOMBIA DURANTE EL PERIODO 2005-2017
}

Rojas Velásquez Libardoํㅜ, Cerquera Losada Oscar Hernán²

Artículo Enviado: 12/09/2017; Aceptado: 17/10/2018; Publicado: 12/02/2019

\section{Resumen}

Este artículo analiza empíricamente las causas de la inflación en Colombia, tomando como punto de referencia las principales escuelas de pensamiento económico que han aportado a este debate. Para ello, se realizan estimaciones econométricas con una muestra de variables económicas y no económicas observadas durante el periodo 2005-2017. Los resultados de las estimaciones muestran que variables macroeconómicas como la masa monetaria, el crecimiento económico, la brecha del producto y la tasa representativa del mercado, tienen un efecto positivo y estadísticamente significativo sobre la inflación. Así mismo, se halla evidencia empírica de la curva de Phillips aumentada y del impacto del fenómeno del niño sobre el nivel general de precios.

Palabras clave: Inflación, nivel de precios, política monetaria, escuelas de pensamiento.

\section{EMPIRICAL ANALYSIS OF THE CAUSES OF INFLATION IN COLOMBIA DURING THE PERIOD 2005-2017}

\begin{abstract}
This article empirically analyzes the causes of inflation in Colombia, take on as a point of reference the main schools of economic's thought have contributed to this debate. Thus, econometric estimations made with a sample of economic and non-economic variables observed during the 2005-2017 period. The results of the estimations show that macroeconomic variables such as the m money supply, economic growth, the product gap and the representative market rate, these have a positive effect and statistically significant on inflation. Likewise, we found empirical evidence of the Phillips curve augmented and the child's phenomenon impact on the general price level.
\end{abstract}

Key words: Inflation, price level, Monetary Policy, Schools of Thought.

\footnotetext{
${ }^{1}$ Estudiante de maestría en economía, Universidad ICESI; economista de la Universidad Surcolombiana

E-mail:librove93@gmail.com.

${ }^{2}$ Magister en economía, Universidad de Buenos Aires; economista Universidad Surcolombiana. Docente de tiempo completo de planta del programa de Economía de la Universidad Surcolombiana. Integrante del grupo de investigación IGUAQUE de la Universidad Surcolombiana, Neiva, Huila, Colombia. E-mail: oscar.cerquera@usco.edu.co
}

ROJAS VELÁSQUEZ, L., \& CERQUERA LOSADA, ÓSCAR H. (2019)

Este es un artículo open Access bajo Licencia Creative Commons Atribución-No Comercial-Sin Derivadas 4.0 Internacional. 


\section{ANALYSE EMPIRIQUE DES CAUSES D'INFLATION EN COLOMBIE AU COURS DE LA PÉRIODE 2005-2017}

\section{Résumé}

Cet article analyse de manière empirique les causes de l'inflation en Colombie, en prenant pour référence les principales écoles de pensée économique qui ont contribué à ce débat. Pour cela, des estimations économétriques sont effectuées avec un échantillon de variables économiques et non économiques observées sur la période 2005-2017. Les résultats des estimations montrent que les variables macroéconomiques telles que la masse monétaire, la croissance économique, l'écart de produit et le taux représentatif de marché ont un effet positif et statistiquement significatif sur l'inflation. De même, il existe des preuves empiriques de la courbe de Phillips augmentée et de l'impact du phénomène de l'enfant sur le niveau général des prix.

Mots clés: inflation, niveau des prix, politique monétaire, écoles de pensée

\section{ANÁLISE EMPÍRICO DAS CAUSAS DA INFLAÇÃO NA COLÔMBIA DURANTE O PERÍODO 2005-2017}

\section{Resumo}

Este artigo analisa empiricamente as causas da inflação na Colômbia, tendo como ponto de referência as principais escolas de pensamento econômico, as quais tem aportado neste debate acadêmico. Por isso realizam-se estimações econométricas, na base de uma mostra de variáveis econômicas e não econômicas observadas durante o período 2005-2017. Os resultados das estimações apresentam variáveis macroeconômicas tais como a taxa monetária, crescimento econômico, a lacuna do produto e a taxa representativa do mercado, tem um efeito positivo e estatisticamente importante sobre a inflação. Deste mesmo modo. acha-se evidência empírica na curva de Phillips, mostrando-se aumentada e do impacto dos fenômenos extratropicais sobre o nível geral dos preços.

Palavras-chave: Inflação. nível de preços, política monetária, escolas de pensamento.

\section{Introducción}

La principal preocupación de las autoridades, tanto políticas como económicas, es mantener la estabilidad y el equilibrio en la economía. Para ello, intentan garantizar un crecimiento económico sostenido, una equitativa distribución de la renta y un nivel de precios moderado. Sin embargo, a pesar de estos esfuerzos, es imposible evitar desajustes en el sistema económico. Con respecto a la inflación, en Colombia se han presentado tres brotes inflacionarios durante los últimos veinte años; el primero a finales del siglo XX debido a la crisis económica mundial (Ochoa Díaz y Martínez Montealegre, 2005); el segundo durante la crisis financiera de 2008 (Cárdenas Pinzón y Vallejo Zamudio, 2012) y el tercero recientemente debido a algunos desajustes estructurales acentuados a raíz de la caída de los precios internacionales del petróleo (Botero García, López Castaño, Posada, Ballesteros Ruíz, y García Guzmán, 2015). En este sentido, la inflación se ha convertido en uno de los fenómenos económicos más conocidos en la actualidad; incluso, en Colombia

ROJAS VELÁSQUEZ, L., \& CERQUERA LOSADA, ÓSCAR H. (2019)

Este es un artículo open Access bajo Licencia Creative Commons Atribución-No Comercial-Sin Derivadas 4.0 Internacional. 
el principal objetivo del Banco Central es garantizar el poder adquisitivo de la moneda nacional ${ }^{3}$ (Cárdenas Pinzón y Vallejo Zamudio, 2012; BANREP, 2016).

Desde la época mercantilista, ha surgido el interés de comprender y explicar el crecimiento del nivel general de precios (Landreth y Colander, 2006). En efecto, las diferentes escuelas de pensamiento, influidas por las circunstancias económicas y políticas, propias de cada época, han intentado dar una explicación coherente acerca del fenómeno de la inflación; no solo con el fin entender sus causas y conocer sus consecuencias, sino con el propósito de reorientar los esfuerzos de la política económica, la cual busca mantener y garantizar el equilibrio económico de las sociedades.

De acuerdo con Jurado Estrada (2005), la teoría cuantitativa atribuye el incremento en el nivel general de precios a factores exclusivamente monetarios. Para la teoría keynesiana la inflación tiene su origen en las desviaciones del producto observado de una economía con respecto a su potencial. Marx (1976) aunque no toca explícitamente el tema de la inflación, plantea que la suma total de los precios de las mercancías, la velocidad promedio del dinero y la masa monetaria utilizada en circulación, son los principales elementos que inciden en el proceso de circulación.

Para el enfoque estructuralista Cepalino, la inflación no es una causa en sí misma, sino una consecuencia de los desajustes estructurales del sistema productivo, esto haciendo referencia a la crisis inflacionaria vivida en Latinoamérica a mediados del siglo XX (Perossa y Waldman, 2015). A este conjunto de teorías se le puede agregar los planteamientos acerca de la curva de Phillips (Phillips, 1958) ${ }^{4}$, la cual estima una relación negativa entre la inflación y el desempleo; sin embargo, en la realidad es muy difícil hallar evidencia empírica de dicha interacción; por tanto, es útil acudir a la curva de Phillips aumentada o la nueva curva de Phillips Neo-Keynesiana, que se ajusta según las expectativas acerca de los precios (Dorbnusch, Fischer, y Startz, 2008; Mendieta y Berbery, 2017). Así las cosas, el incremento del nivel de precios puede estar influido por factores de largo plazo, como, el exceso de dinero, las políticas inconsistentes y los déficits fiscales; o por factores de corto plazo, como, contracciones de la oferta agregada, incrementos de la demanda agregada, la tasa de interés, las expectativas y las externalidades.

En este contexto, el presente ejercicio investigativo analiza empíricamente el fenómeno de la inflación en Colombia durante el periodo 2005-2017, a través de un análisis estadístico y la estimación causal de un modelo de regresión lineal por el método de Mínimos Cuadrados Ordinarios (MCO), con el fin de identificar las principales causas del incremento sostenido del nivel general de precios en la economía colombiana, para luego contrastar estos resultados con los soportes teóricos y empíricos más importantes desarrollados en torno a esta materia.

\section{Marco Teórico y Conceptual}

De acuerdo con Mímica (2007), la inflación es: "el incremento generalizado de los precios de los bienes y de los servicios durante un período de tiempo prolongado, que produce como consecuencia un descenso del valor del dinero y, por tanto, de su poder adquisitivo" (pág. 2). Esta definición es similar a la de Bernanke y Frank (2007) que conceptualizan la inflación como la tasa a la que suben con el paso del

\footnotetext{
${ }^{3}$ El control de la inflación es el principal propósito de la política monetaria en Colombia mediante la estrategia de inflación objetivo implementada por el Banco de la Republica

${ }^{4}$ En 1958, el economista William Phillips, publicó un artículo titulado "La relación entre el desempleo y la tasa de variación de los salarios monetarios en el Reino Unido, 1861-1957", donde demostraba que durante este periodo se produjo una correlación negativa entre la tasa de desempleo y la variación de los salarios en la economía británica.
}

ROJAS VELÁSQUEZ, L., \& CERQUERA LOSADA, ÓSCAR H. (2019)

Este es un artículo open Access bajo Licencia Creative Commons Atribución-No Comercial-Sin Derivadas 4.0 Internacional. 
tiempo los precios en general. Lo anterior implica que la inflación es una medida del aumento del promedio generalizado de los precios de una canasta de consumo representativa. Los referentes conceptuales en torno a la inflación hacen hincapié en el factor tiempo; por tanto, el aumento del promedio general de los precios debe ser permanente (sostenido) y no transitorio, como sucede en algunos casos.

Según postulados de Dornbusch, Fischer y Startz (2008), la inflación es la tasa de cambio de los precios, la cual puede expresarse de la siguiente manera:

$$
\pi=\frac{P_{t}-P_{t-1}}{P_{t-1}}
$$

Donde $\pi$ representa la tasa de inflación, $P_{t}$ el nivel de precios actual y $P_{t-1}$ el nivel de precios del periodo inmediatamente anterior.

Conocer la tasa de cambio de los precios implica necesariamente desarrollar formas de medir el comportamiento de los precios a lo largo del tiempo. El instrumento básico empleado por los economistas para calcular la inflación es el índice de precios, más específicamente el Índice de Precios al Consumidor $(\mathrm{IPC})^{5}$; Sin embargo, se pueden distinguir en este contexto otros índices de precios, como por ejemplo el deflactor del $\mathrm{PIB}^{6}$, el Índice de Precios al Productor $(\mathrm{IPP})^{7}$, el deflactor de gasto de consumo personal $(\mathrm{GCP})^{8}$, así como índices de la inflación subyacente básica $^{9}$.
Según Dornbusch, Fisher y Startz (2008), "el IPC mide el costo de comprar una canasta fija de bienes y servicios que representa las compras de los consumidores urbanos" (pág. 41); mientras que para Bernanke y Frank (2007), "el IPC de un periodo cualquiera mide el coste que tiene en ese periodo una cesta normalizada de bienes y servicios en relación con el coste que tenía esa misma cesta en un determinado año, llamado año base" (pág. 54). De esta manera, el IPC es el cociente entre el coste de una cesta de bienes y servicios del año base en el año actual y el coste de esa misma cesta del año base en el año base; por tanto, el IPC se constituye en uno de los instrumentos más utilizados para calcular la inflación. Es necesario tener en cuenta que por lo general este indicador presenta una tendencia creciente con el paso del tiempo.

\section{Teorías económicas acerca de la inflación}

La inflación es claramente un fenómeno económico acentuado en la actualidad; sin embargo, el interés por el estudio del incremento del nivel general de los precios se originó aún en los propios inicios de la teoría económica. Landreth y Colander (2006), sitúan los primeros estudios acerca de la inflación durante la época mercantilista, cuando los economistas de la época notaron un incremento de los precios en Europa debido a la entrada de metales preciosos provenientes del nuevo mundo. En efecto, desde entonces se han venido haciendo una serie de aportes teóricos y empíricos que han consolidado una teoría formal en torno a la inflación. Las teorías económicas acerca de

\footnotetext{
${ }^{5}$ En Colombia el cálculo del IPC es responsabilidad del DANE, institución que mensualmente publica los resultados del indicador para el mes inmediatamente anterior.

${ }^{6}$ Es el indicador más general de la inflación y deflación, pues considera todos los precios de bienes y servicios finales producidos en una economía. ${ }^{7} \mathrm{El}$ IPP es un indicador de la evolución de los precios de venta del productor calculado por el DANE, correspondientes al primer canal de comercialización o distribución de los bienes transados en la economía.

${ }^{8}$ Es un indicador económico de Estados Unidos que mide la inflación de forma similar al IPC. Este índice mide el incremento medio de los precios de bienes y servicios de consumo personal, pero a diferencia del IPC, no utiliza una canasta representativa fija, sino que el índice cambia junto con los hábitos de gasto del consumidor.

${ }^{9}$ Es un indicador que mide la variabilidad de los precios de consumo a corto plazo excluyendo del IPC los componentes más volátiles como el índice energético y el índice de alimentos no elaborados.
}

ROJAS VELÁSQUEZ, L., \& CERQUERA LOSADA, ÓSCAR H. (2019)

Este es un artículo open Access bajo Licencia Creative Commons Atribución-No Comercial-Sin Derivadas 4.0 Internacional. 
la inflación han tratado de interpretar y explicar este fenómeno, partiendo de las concepciones fundamentales de las distintas escuelas de pensamiento económico, las cuales han estado influidas por una serie de problemáticas, intereses y circunstancias históricas específicas.

\subsection{Teoría cuantitativa}

Partiendo de los aportes de Jurado Estrada (2005), en la teoría cuantitativa el poder adquisitivo del dinero depende directamente de su cantidad; por tanto, un aumento de la cantidad de dinero en circulación implica necesariamente un detrimento en el valor de la moneda. Esto se debe a que un incremento de la oferta monetaria conlleva también a un incremento de la demanda de bienes y servicios, lo que a su vez estimula un alza en el promedio general de los precios, dando lugar a una pérdida de valor del dinero. De esta manera, se puede afirmar que el nivel de precios depende de la oferta monetaria. En este sentido, la teoría cuantitativa puede expresarse de la siguiente manera:

$$
M=K P \quad \text { o } \quad P=\frac{1}{K M}
$$

Donde $M$ representa la cantidad de dinero, $K$ es un factor de proporcionalidad que permanece constante y $P$ el nivel general de precios. La teoría cuantitativa presenta dos versiones o aproximaciones teóricas. Por un lado, la ecuación de cambio de Irving Fisher, conocida también como el "método de transacciones"; la cual incluye nuevos factores, como la velocidad de circulación del dinero y el número de operaciones. Por tanto, puede expresarse así:

$$
P T=M V
$$

Donde se establece una conexión importante entre la oferta monetaria $(M)$, la velocidad del dinero $(V)$, el volumen de transacciones $(T)$, y el nivel de precios $(P)$. De acuerdo con esta identidad, se dice que el valor de todas las ventas es igual al valor de todas las compras. Así mismo, se puede decir que: "el nivel de precios será proporcional a la oferta monetaria cuando se supone que la velocidad del dinero está determinada por factores institucionales en el sector monetario (...) y si se considera que el sector real determina el volumen de transacciones" (Jurado Estrada, 2005, pág. 5). En este sentido, el nivel de precios se expresa mediante la siguiente ecuación:

$$
P=\left(\frac{V}{T}\right) M
$$

Por otro lado, la ecuación cuantitativa de la escuela de Cambridge, conocida también como "la teoría de los saldos monetarios", la cual parte de principios microeconómicos para explicar que las demandas individuales de dinero pueden agregarse en una demanda macroeconómica, proporcional al nivel de renta nominal. De esta manera, a través de la teoría de los saldos monetarios reales se puede demostrar que la tasa de crecimiento de la oferta monetaria $(m)$, es resultado de la suma de la tasa de crecimiento del producto nacional $(y)$, tomado en términos reales, $\mathrm{y}$ la tasa de inflación $(p)$, tal y como se muestra en la siguiente ecuación:

$$
m=y+p
$$

Por tanto, la tasa de inflación es igual a la diferencia entre la tasa de crecimiento de la oferta monetaria y la tasa de crecimiento del producto nacional.

\subsection{Teoría Keynesiana}

La teoría keynesiana nace en el marco de la crisis económica vivida en la década de los 30's del siglo pasado. Por tanto, Jurado Estrada (2005), tomado como referencia a Brand (1987), asegura que: "La Teoría General de Keynes, se funda en un discurso teórico explicativo de las medidas de política económica que los gobiernos deben adoptar para superar las limitaciones de la teoría económica clásica, y salvar al mundo capitalista de la grave recesión" (pág. 7). En este sentido, Keynes se aleja un

ROJAS VELÁSQUEZ, L., \& CERQUERA LOSADA, ÓSCAR H. (2019)

Este es un artículo open Access bajo Licencia Creative Commons Atribución-No Comercial-Sin Derivadas 4.0 Internacional. 
poco de los tradicionales postulados monetaristas, priorizando supuestos relacionados con la demanda efectiva; sin embargo, este tipo de demanda se constituye como uno de los estimulantes directos del nivel general de precios.

Según los conceptos introducidos por los keynesianos, el crecimiento de la demanda agregada crea presiones inflacionarias siempre y cuando la economía emplee plenamente los recursos disponibles; puesto en otras palabras, cuando la producción real sea igual a la producción potencial ${ }^{10}$. Por tanto, es poco probable que haya inflación cuando hay subutilización de recursos en la economía. Keynes desarrolla sus aportes teóricos en esta materia partiendo de las experiencias inflacionarias de algunos países, sobre todo de Europa, a través de su conocido modelo de la brecha inflacionaria. En este modelo parte de que la demanda real corriente está constituida por la demanda de consumo real y la demanda de inversión real, de la siguiente manera:

$$
Y_{t}=C_{t}+I_{t}=Y_{0}
$$

Donde $Y_{t}$ es la demanda real corriente, $C_{t}$ el consumo real, $I_{t}$ la inversión real y $Y_{0}$ es la producción real de pleno empleo. Entonces si $Y_{t}=Y_{0}$ la producción real es igual a la producción potencial. El consumo real se expresa de la siguiente manera:

$$
C_{t}=a+b \frac{Y P_{t-1}}{P_{t}}
$$

Donde $a$ es el consumo autónomo real, $b$ la propensión marginal al consumo, $Y P_{t-1}$ el ingreso monetario del periodo anterior y $P_{t}$ el nivel de precios corriente. Además, se supone que la inversión real es exógena y que el producto entre la demanda real corriente y el nivel de precios corriente es igual al producto entre la producción real de pleno empleo y el nivel de precios corriente. Por tanto, sustituyendo y realizando algunos cálculos se obtiene que:

$$
\begin{gathered}
\frac{P_{t}}{P_{t-1}}=\frac{b Y}{Y_{0}-\left(a+I_{0}\right)} \\
P_{t}=\frac{\left[\left(a+I_{0}\right)+b Y_{0}\right]-Y_{0}}{Y_{0}-\left(a+I_{0}\right)}
\end{gathered}
$$

En este sentido, el numerador del lado derecho de (9) se considera como la brecha inflacionaria. Cuanto más grande sea esta brecha mayor será la tasa de inflación. Por tanto, la inflación surge de una desviación de la demanda agregada por encima de la capacidad de pleno empleo de la economía.

\subsection{Concepción marxista}

Si bien es cierto, la inflación no es un tema caracterizado dentro de los principales postulados marxistas, Marx si desarrolla una explicación acerca del dinero dentro del sistema económico mediante la ley de circulación, expuesta en su obra El Capital. En este sentido, Jurado Estrada (2005) tomando las ideas de Marx (1867) afirma que: "la cantidad de dinero necesaria para asegurar la circulación de mercancías debe equivaler a la suma de los precios de todas las mercancías, divididas por el promedio de ciclos de la circulación de una unidad monetaria del mismo signo" (pág. 10). Lo anterior puede expresarse en términos matemáticos de la siguiente manera:

$$
M=\frac{\sum P M}{V}
$$

Donde $M$ es la cantidad de dinero en circulación, $P M$ los precios de las mercancías y $V$ la velocidad de circulación. De acuerdo a esto, se puede decir que, para Marx, la suma total de los precios de las mercancías, la velocidad promedio de circulación del dinero y la masa monetaria utilizada en circulación, son los principales elementos que inciden en el proceso de circulación. Según diversos aportes teóricos, en la ley marxista de la circulación se invierte la hipótesis de la teoría

10 Producto a que puede llegar la economía si hay una situación de pleno empleo de los recursos.

ROJAS VELÁSQUEZ, L., \& CERQUERA LOSADA, ÓSCAR H. (2019)

Este es un artículo open Access bajo Licencia Creative Commons Atribución-No Comercial-Sin Derivadas 4.0 Internacional. 
cuantitativa, porque la velocidad de circulación y los precios de las mercancías se convierten en los determinantes del volumen de dinero en el mercado. En cuanto a esto, Consuegra (2000) afirma que: En la Ley de Marx son los precios, la cantidad de mercancías ofrecidas y el ritmo de circulación, los que determinan la masa monetaria" (pág. 105)

\subsection{Enfoque estructuralista}

Debido al fenómeno inflacionario presentado en América Latina en los años 50's del siglo XX, un grupo de economistas de la región agrupados en torno a la Comisión Económica Para América Latina y el Caribe (CEPAL), plantearon una serie de principios dirigidos a interpretar y controlar esta situación. En este contexto surge la concepción estructuralista de la inflación, la cual destaca las características de la estructura productiva de Latinoamérica. Estas premisas se contraponen al enfoque exclusivamente monetarista de órganos económicos internacionales como el Fondo Monetario Internacional (FMI).

Para la concepción estructuralista, "la inflación y otros desequilibrios de la economía son manifestaciones y no causas de las crisis económicas, las causas, por lo tanto, deben ubicarse en las estructuras de producción, distribución y consumo de la sociedad" (Jurado Estrada, 2005, pág. 13). Según estos postulados, puede existir una relación positiva entre la tasa de inflación y la tasa de crecimiento económico, al menos hasta cierto punto. Entonces, para el estructuralismo la estabilidad de precios se obtendrá solo a través del crecimiento económico. Así las cosas, las causas de la inflación son estructurales, mientras que los aspectos monetarios son catalogados únicamente como propagadores de la inflación (Perossa y Waldman, 2015). Así, el fenómeno inflacionario en Latinoamérica es caracterizado en dos categorías: los factores estructurales y los mecanismos de propagación.

Los factores estructurales hacen referencia a la insuficiencia en el crecimiento económico debido a la ineficiencia productiva en algunos sectores de la economía donde la demanda presenta un comportamiento inelástico. En efecto, el precario desarrollo y el tamaño de las economías latinoamericanas impiden satisfacer completamente la demanda interna, por lo que se genera una fuerte dependencia del exterior; por tanto, cuando los precios internacionales se incrementan se da lugar a presiones inflacionarias en estas economías. Así mismo, las dificultades originadas en torno a la propiedad y la explotación de la tierra hacen que la oferta agrícola sea muy rígida; de hecho, Jurado Estrada (2005) afirma que: "la producción agrícola es muy inelástica respecto a los precios, el crecimiento de la población urbana en Latinoamérica y el consecuente aumento de la demanda de productos alimenticios y agrícolas no se traducen por tanto en aumentos de producción sino en aumentos de precios" (pág. 14)

\subsection{Causas de la inflación}

Los determinantes de la inflación pueden agruparse de acuerdo al horizonte de tiempo en que estos tarden en impactar los precios. En este sentido, el Banco Central de México (2012) distingue entre determinantes de largo plazo y determinantes de corto plazo. Dentro de los determinantes de largo plazo, se pueden distinguir tres factores: el exceso de dinero, el déficit fiscal y las políticas inconsistentes (De Gregorio, 2000). Por su parte, dentro de los determinantes de corto plazo de la inflación se pueden distinguir cuatro factores: la contracción de la oferta agregada, el incremento de la demanda agregada, la tasa de interés y las expectativas de inflación.

Mímica (2007), menciona tres fuentes de inflación, reforzando los criterios expuestos en el acápite anterior; para este autor existe una inflación de demanda, que se da cuando la demanda general de bienes aumenta sin que el sector productivo se haya adaptado a este cambio; una inflación de costos, que tiene lugar cuando el costo de producción se incrementa, entonces los productores en su afán de mantener sus utilidades

ROJAS VELÁSQUEZ, L., \& CERQUERA LOSADA, ÓSCAR H. (2019)

Este es un artículo open Access bajo Licencia Creative Commons Atribución-No Comercial-Sin Derivadas 4.0 Internacional. 
suben los precios; y una inflación auto construida, ligada al hecho de que los agentes prevén aumentos futuros de precios y ajustan su conducta actual a esa previsión futura, esto es lo que se conoce como expectativas sobre la inflación.

En este contexto es necesario tener en cuenta como causales de inflación a los factores externos que crean choques de oferta o de demanda (Avella, 2001; Abril Salcedo, Melo Velandia, y Parra Amado, 2016). Dichos factores externos tienen un efecto de trasmisión similar a los determinantes ya descritos, debido a que generan alteraciones tanto en la oferta agregada como en la demanda agregada, especialmente a través de variaciones en el IPC de los alimentos. Por ejemplo, un choque externo puede causar un decremento de la oferta o estimular la demanda; por ende, los precios van a presentar un comportamiento alcista. La diferencia radica en que los factores externos no tienen su origen en el campo económico, sino que pueden ser explicados por afectaciones naturales, políticas o por cambios en las preferencias de los agentes en la economía.

\subsection{Curva de Phillips}

A partir del estudio desarrollado por Phillips (1958), acerca del comportamiento de los salarios en el Reino Unido entre 1861 y 1957, se logró establecer una relación negativa entre la tasa de inflación en los salarios y el desempleo. En este contexto, Dornbusch, Fischer y Startz (2008) afirman que: "la curva de Phillips es una relación inversa entre la tasa de desempleo y el ritmo a que aumentan los salarios en efectivo. Cuando más elevada es la tasa de desempleo, menor es la tasa de inflación salarial" (pág. 120). Así, la tasa de inflación salarial se expresa como:

$$
g_{w}=\frac{W_{t+1}-W_{t}}{W_{t}}
$$

Donde $g_{w}$ es la tasa de inflación de los salarios, $W_{t}$ el salario del periodo actual y $W_{t+1}$ el salario de un periodo siguiente. De este modo, la curva simple de Phillips puede expresarse también como:

$$
g_{w}=-\in(u-u *)
$$

Donde $\in$ mide la sensibilidad de los salarios al desempleo, $u$ es la tasa de desempleo y $u^{*}$ la tasa natural de desempleo ${ }^{11}$. Así, "esta ecuación establece que los salarios bajan cuando la tasa de desempleo excede la tasa natural (...) y suben cuando el desempleo es menor que dicha tasa", (Dorbnusch, Fischer, \& Startz, 2008, pág. 121). Por ejemplo, suponiendo que en una economía se presenta una tasa de desempleo igual a la tasa natural y un nivel de precios estable, si se produce un aumento en las reservas de dinero en una determinada magnitud, entonces los precios y los salarios deberán aumentar en la misma proporción para que la economía esté de nuevo en equilibrio; sin embargo, de acuerdo con los postulados tomados a partir de la curva de Phillips, para que los salarios suban en dicha cantidad es necesario que la tasa de desempleo baje.

A pesar de la importancia de este concepto teórico en el análisis económico, la realidad es que el supuesto de la curva de Phillips se desmoronó después de la década de los 60's, porque se observó que los datos reales de aquellas épocas no correspondían a la explicación de esta teoría. Es así como a la simple curva de Phillips se le añadieron fundamentos microeconómicos mediante la inclusión de una medida de la inflación esperada o anticipada. En efecto, la relación entre inflación y desempleo ya no depende del nivel general de precios, sino del monto en que estos superan las expectativas. Es decir que, ahora el interés está centrado en el aumento de los salarios por encima de la inflación' esperada. Por lo tanto, la curva de

\footnotetext{
11 Es aquella tasa que representa un nivel de desempleo que no puede ser reducido y forma parte de lo que se considera normal en una economía. Es la tasa de paro de equilibrio en el largo plazo.
}

ROJAS VELÁSQUEZ, L., \& CERQUERA LOSADA, ÓSCAR H. (2019)

Este es un artículo open Access bajo Licencia Creative Commons Atribución-No Comercial-Sin Derivadas 4.0 Internacional. 
Phillips aumentada o la nueva curva de Phillips-Neokeynesiana, como también se le conoce, puede expresarse como:

$$
\left(g_{w}-\pi^{e}\right)=-\in(u-u *)
$$

Donde $\pi^{e}$ es el nivel de inflación esperada de los precios. De acuerdo con Dornbusch, Fischer y Startz (2008), si se mantiene la suposición de un salario real constante, la inflación real, $\pi$, es igual a la inflación salarial. Así, la ecuación de la nueva curva de Phillips aumentada con expectativas puede expresarse de la siguiente manera:

$$
\pi=g_{w}=\pi^{e}-\in(u-u *)
$$

\section{Metodología}

Con el propósito de analizar empíricamente las causas de la inflación en Colombia, se consolidó una base de datos con un grupo de variables de interés sugeridas por la literatura (Phillips, 1958; De Gregorio, 2000; Jurado Estrada, 2005; Mimica, 2007; Banco de Mexico, 2012; Abril Salcedo, Melo Velandia, y Parra Amado, 2016).

Las variables corresponden a algunos de los principales agregados macroeconómicos como la masa monetaria, la tasa de interés, la Tasa Representativa del Mercado (TRM), las tasas de empleo y desempleo, el déficit fiscal, las importaciones, el Producto Interno Bruto (PIB), la brecha observada entre el PIB real y el PIB potencial y las expectativas sobre la inflación. Adicionalmente, se incluyeron variables binarias ${ }^{12}$ para caracterizar externalidades negativas originadas a partir de fenómenos naturales ${ }^{13}$.

Con base en estos datos se realizaron estimaciones econométricas mediante $\mathrm{MCO}$, con el ánimo de explicar los cambios de la variable dependiente o explicada $\left(y_{t}\right)$, en este caso la tasa de inflación, en función de las variables independientes o explicativas $\left(x_{1 t}, x_{2 t}, \ldots, x_{k t}\right)$, como se observa en $(15)^{14}$.

$$
y_{t}=\beta_{0}+\beta_{1} x_{1 t}+\beta_{2} x_{2 t}+\ldots+\beta_{k} x_{k t}+\mu_{t}
$$

Donde $\beta_{0}$ es el intercepto y $\beta_{1}, \beta_{2}, \ldots, \beta_{k}$ son parámetros que miden los cambios en la variable dependiente, respecto a cada una de las variables explicativas, manteniendo los demás factores constantes, es decir que estos coeficientes representan la pendiente de la función de regresión. Por su parte, $\mu_{t}$ es el termino de error o perturbación inobservable que contiene factores distintos a $x_{1 t}, x_{2 t}, \ldots, x_{k t}$ que afectan a $y_{t}$.

De acuerdo con Wooldridge (2010), el método de MCO minimiza la suma de los cuadrados de las distancias verticales entre los valores de los datos y los de la regresión estimada, es decir que, minimiza la suma de los cuadrados del residuo. Por tanto, si se cumplen todos los supuestos del modelo clásico de regresión lineal, los parámetros estimados por MCO son los Mejores Estimadores Lineales Insesgados (MELI). El análisis empírico consistió en observar la magnitud y el sentido de los parámetros estimados, validando su significancia estadística a través de pruebas de hipótesis con niveles de confiabilidad de $90 \%, 95 \%$ y $99 \%$.

\section{Comportamiento de la inflación en Colombia}

La economía colombiana presentó un fuerte choque inflacionario a finales del siglo pasado, debido a la crisis económica mundial. A partir de entonces la inflación se mantuvo en cifras moderadas. Fue hasta el año 2008, en el escenario de la crisis financiera global, cuando la

\footnotetext{
12 Variables que toman valores restringidos que pueden ser 0 o 1

${ }^{13}$ Fenómeno del niño y de la niña

${ }^{14} \mathrm{El}$ subíndice $\mathrm{t}$ indica que las variables se observan a través del tiempo.
}

ROJAS VELÁSQUEZ, L., \& CERQUERA LOSADA, ÓSCAR H. (2019)

Este es un artículo open Access bajo Licencia Creative Commons Atribución-No Comercial-Sin Derivadas 4.0 Internacional. 
inflación de nuevo se convirtió en preocupación para las autoridades monetarias, sin embargo, desde el año 2009 este indicador mostró una tendencia decreciente. No obstante, debido a diversas circunstancias, tanto económicas como no económicas, el nivel general de precios empezó a mostrar un comportamiento alcista desde 2014, tal y como puede verse en el gráfico 1. Por tanto, la inflación se convirtió de nuevo en una de las principales problemáticas del quehacer de la política económica en Colombia. Sin embargo, la variación del IPC ha vuelto a encausarse hacia su rango meta a partir de 2017. De igual manera, el gráfico 1 muestra que la variación mensual del IPC en Colombia presenta comportamientos cíclicos y estacionales.
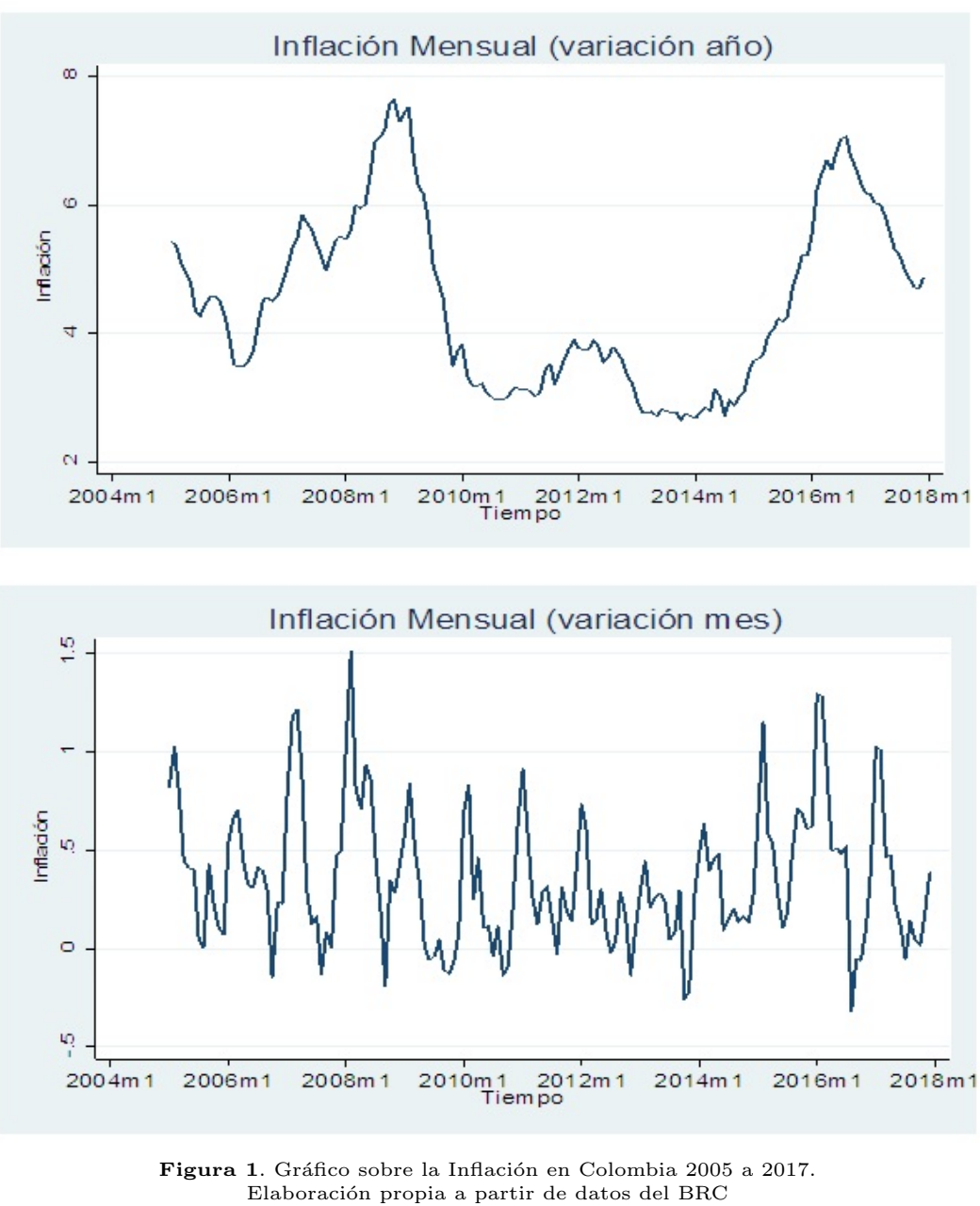

De acuerdo con el Banco de la Republica (2016), en Colombia, "La política monetaria (...) se rige por un esquema de meta de inflación, en el cual el objetivo principal es alcanzar tasas bajas de inflación y buscar la estabilidad del crecimiento del producto alrededor de su tendencia de largo plazo" (pág. 4). En la actualidad la inflación objetivo corresponde al $3 \%$, con una banda que establece un límite inferior en $2 \%$ y un límite superior en $4 \%{ }^{15}$. El ajuste de la tasa de inflación hacia su rango meta puede observarse en el gráfico 2. Así,

\footnotetext{
15 El esquema de inflación objetivo de manera gradual a partir de 1991, cuando se le otorgó independencia al Banco de la República. Para ver detalles acudir a Cárdenas Pinzón y Vallejo Zamudio (2012).
}

ROJAS VELÁSQUEZ, L., \& CERQUERA LOSADA, ÓSCAR H. (2019)

Este es un artículo open Access bajo Licencia Creative Commons Atribución-No Comercial-Sin Derivadas 4.0 Internacional. 
durante las épocas de alta inflación, como la de 2008 o la de 2016, los precios en Colombia se alejaron de su objetivo, aunque estuvieron dentro de estas fronteras entre 2010 y mediados de 2014.

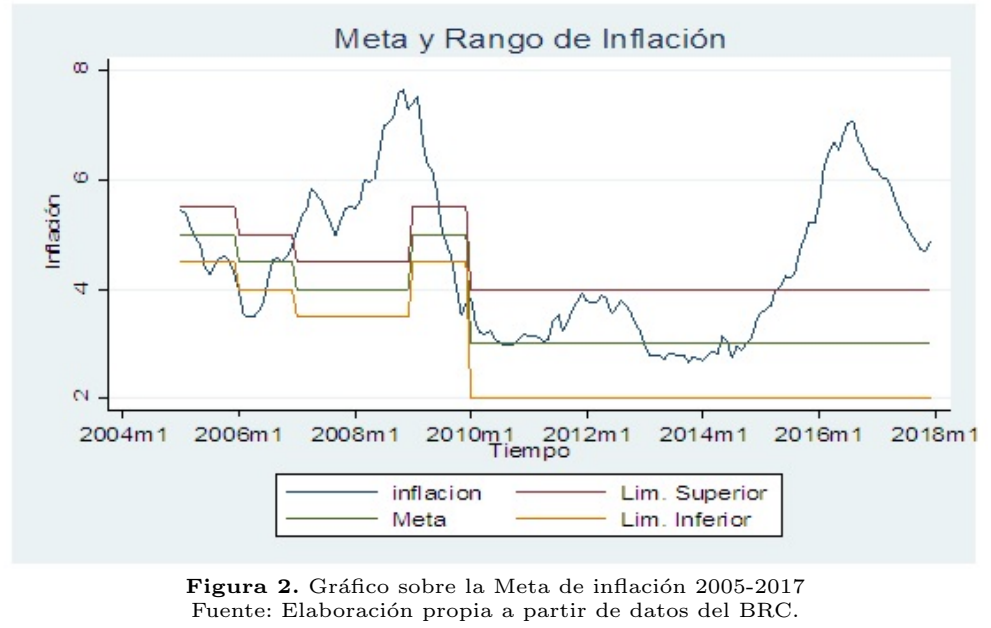

En lo que respecta a las expectativas sobre la inflación, el gráfico 3, muestra que la tasa real de variación del IPC tiende a ajustarse a la tasa esperada; sin embargo, los mayores desfases se observaron en los periodos donde la inflación creció vertiginosamente. La

literatura económica sugiere que una de las principales causas del crecimiento de la inflación se debe al comportamiento de las expectativas, dado que los agentes económicos pueden prever el incremento de los precios y ajustar sus decisiones de consumo y ahorro.

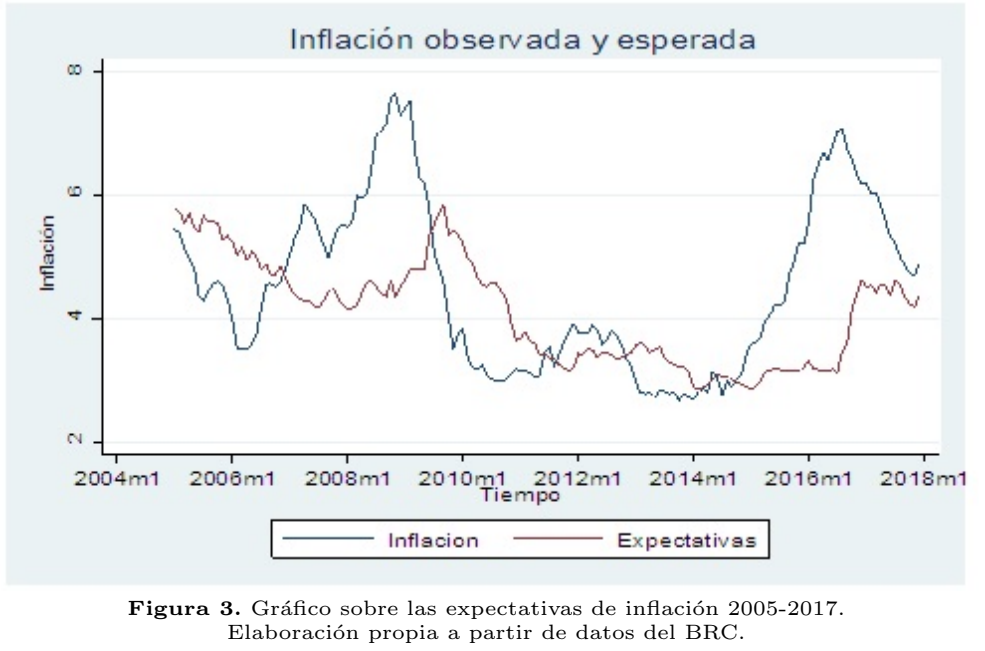

Analizando el comportamiento del nivel general de precios por grupo de gasto (véase gráfico 4), se halló que para el periodo 2005-2017, el precio de la canasta de alimentos fue muy volátil en la economía colombiana; incluso, superó los niveles de los demás grupos de gasto.
Por su parte, los precios del vestuario, la diversión y las comunicaciones permanecieron relativamente bajos. Los precios del transporte, la educación, la salud y los correspondientes a otros grupos de gasto se comportaron de manera muy similar al promedio general.

ROJAS VELÁSQUEZ, L., \& CERQUERA LOSADA, ÓSCAR H. (2019)

Este es un artículo open Access bajo Licencia Creative Commons Atribución-No Comercial-Sin Derivadas 4.0 Internacional. 

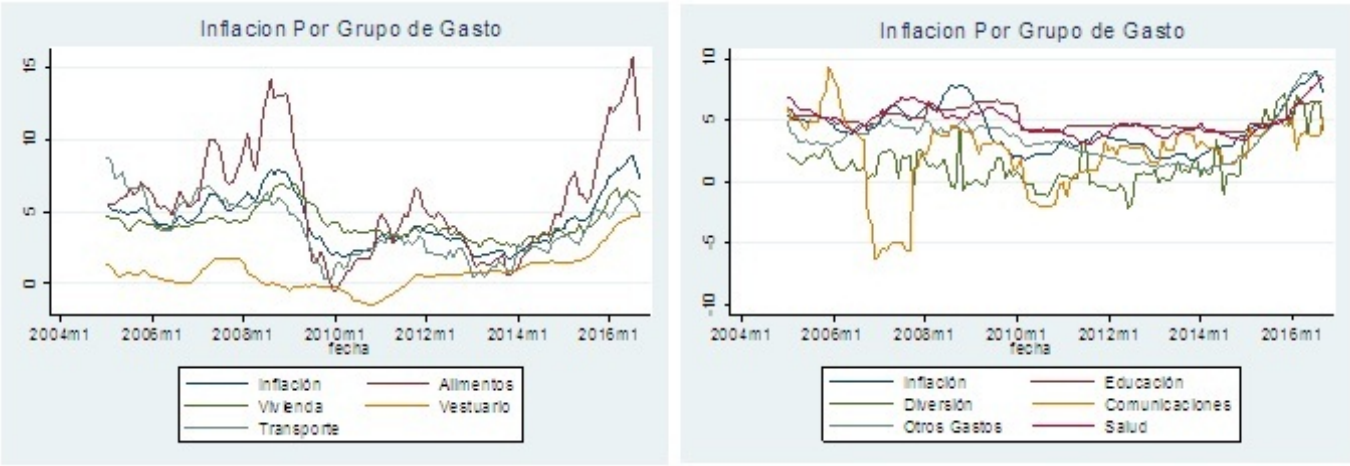

Figura 4. Gráfico sobre la Inflación por grupos de gasto 2005-2016 Fuente: Elaboración propia a partir de datos del BRC

En lo referente al análisis de la inflación por ciudades (véase gráfico 5), dentro de las ciudades principales, Barranquilla fue la que presentó mayor nivel general de precios durante el periodo observado, mientras que la inflación más baja se halló en la ciudad de Cali. En Bogotá y Medellín se observó un comportamiento similar al promedio.
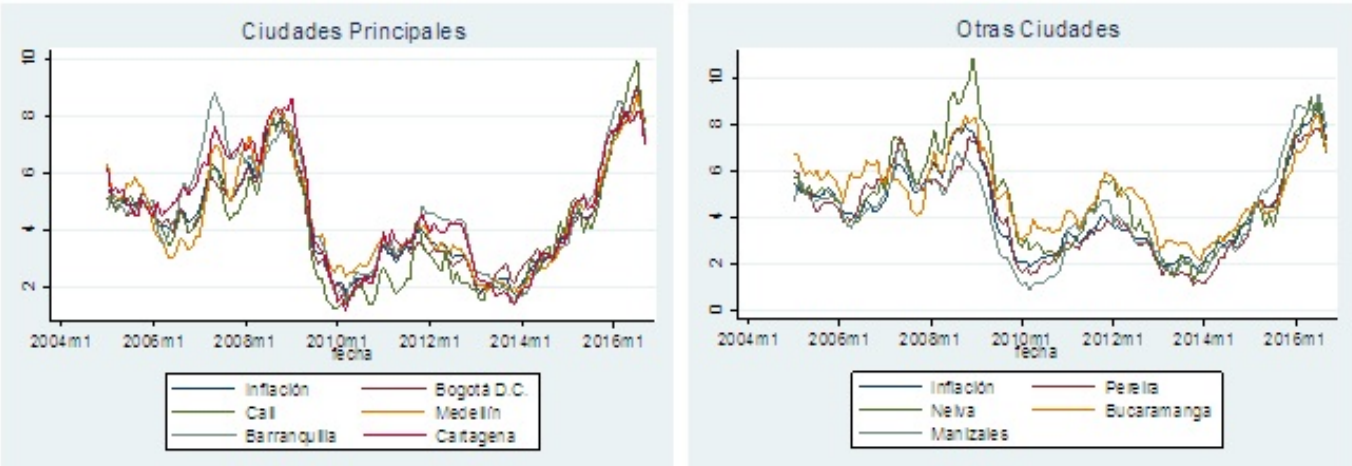

Figura 5. Gráfico sobre la Inflación por ciudades 2005-2016 Fuente: Elaboración propia a partir de datos del BRC

En cuanto a las demás ciudades, se puede observar que los precios fueron más altos en Neiva y Bucaramanga, sobre todo durante el periodo de la crisis financiera mundial; los precios más bajos tuvieron lugar en Manizales; mientras que Pereira se ubicó en torno al promedio nacional de los cambios en el IPC.

\section{Resultados y Discusión}

Tomando como referente los postulados teóricos analizados anteriormente, se realizaron estimaciones econométricas orientadas a identificar las causas de la inflación en Colombia a través de la evidencia empírica. Debido a la dificultad para la consolidación y medición de los datos estadísticos a través del tiempo, se plantearon dos modelos de regresión, el primero tiene en cuenta variables con periodicidad mensual, mientras que en el segundo modelo se incluyen datos de periodicidad trimestral. En este acápite es útil aclarar que algunas variables explicativas como el flujo de importaciones y la tasa de empleo no fueron incluidas en las estimaciones finales debido a que estaban asociadas a problemas de multicolinealidad por su alto grado de correlación con otras variables, generando de esta manera un sesgo en los cálculos por MCO. De igual manera, la variable que representa el déficit fiscal y el fenómeno de la niña no se incluyeron en los resultados finales porque carecen de significancia

ROJAS VELÁSQUEZ, L., \& CERQUERA LOSADA, ÓSCAR H. (2019)

Este es un artículo open Access bajo Licencia Creative Commons Atribución-No Comercial-Sin Derivadas 4.0 Internacional. 
estadística, y por tanto son inútiles para hacer contrastaciones empíricas.

Como se observa en la tabla 1, las estimaciones realizadas con datos de periodicidad mensual presentan una bondad de ajuste de 87,59\%, mientras que en las estimaciones realizadas con datos de periodicidad trimestral presentan un coeficiente de determinación equivalente a 86,63\%; esto indica que las variables independientes seleccionadas para controlar los cambios en la tasa de inflación, tienen un poder explicativo superior al $80 \%$. Así mismo, la prueba F muestra que los dos modelos econométricos son globalmente significativos en términos estadísticos.

En este contexto, la oferta de dinero tiene un efecto positivo sobre la inflación, porque un incremento de
$1 \%$ en la masa monetaria conduce a un aumento de la inflación de 1,22\%. De igual modo, la tasa de interés tiene un efecto positivo sobre el nivel de precios, porque un aumento de una unidad porcentual en la tasa de intervención provoca un incremento de 0,57 puntos porcentuales en la tasa de cambio de los precios; sin embargo, de acuerdo con los aportes teóricos, este resultado es contra intuitivo, dado que, cuando suben los intereses se reduce la demanda agregada desincentivando la inversión y el consumo, aumentando el ahorro de las personas; de esta manera se limita la cantidad de dinero disponible en la economía con lo que el nivel de precios disminuye; no obstante, esta distorsión puede estar explicada por el hecho de que algunos brotes inflacionarios en la economía colombiana se han dado a través de canales de oferta (Ochoa Díaz y Martínez Montealegre, 2005).

Tabla 1. Resultado de las estimaciones econométricas

\begin{tabular}{|c|c|c|}
\hline \multirow[t]{2}{*}{ Variables explicativas } & \multicolumn{2}{|c|}{ Variables dependiente } \\
\hline & Inflación mensual & Inflación trimestral \\
\hline Masa monetaria & $\begin{array}{c}1,2204^{* * *} \\
(0,175)\end{array}$ & - \\
\hline Tasa de interes & $\begin{array}{c}0,5733^{* * *} \\
(0,025)\end{array}$ & $\bar{L}$ \\
\hline TRM & $\begin{array}{c}1,1698^{* * *} \\
(0,305)\end{array}$ & 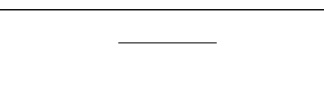 \\
\hline Desempleo & $\begin{array}{c}0,1257^{* * *} \\
(0,035)\end{array}$ & 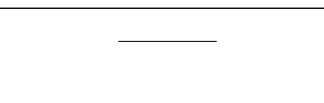 \\
\hline Expectativas & $\begin{array}{c}0,3392^{* * *} \\
(0,078)\end{array}$ & 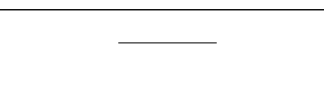 \\
\hline Fenómeno del niño & $\begin{array}{l}0,258^{* * *} \\
(0,103)\end{array}$ & $\underline{-}$ \\
\hline Crecimiento económico & - & $\begin{array}{c}0,1349^{* * *} \\
(0,040)\end{array}$ \\
\hline Brecha del producto & 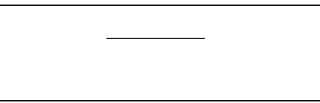 & $\begin{array}{c}3,8176^{\text {*** }} \\
(0,349)\end{array}$ \\
\hline Constante & $\begin{array}{c}-25,6443^{* * *} \\
(2,470)\end{array}$ & $\begin{array}{c}-3,8138^{* * *} \\
(0,648)\end{array}$ \\
\hline R-squaed & 08759 & 0.8663 \\
\hline Prob $>F$ & 0,0000 & 0,0000 \\
\hline
\end{tabular}

Nota: ${ }^{* * *}$ p-value $<0,01 ;{ }^{* *}$ p-value $<0,05$.

Entre paréntesis, el error estándar.

Fuente: Cálculos propios a partir de datos del BRC, DANE y el IDEAM

ROJAS VELÁSQUEZ, L., \& CERQUERA LOSADA, ÓSCAR H. (2019)

Este es un artículo open Access bajo Licencia Creative Commons Atribución-No Comercial-Sin Derivadas 4.0 Internacional. 
La TRM incrementa el promedio del nivel general de precios, debido a que un incremento de $1 \%$ en el tipo de cambio está asociado a un aumento de la inflación equivalente a 1,16\%. Efectivamente, antes de la crisis petrolera de 2014 el promedio de la TRM se ubicaba alrededor de $\$ 1800$, mientras que para finales de 2016 se ubicó alrededor de $\$ 3100$, un incremento de $\$ 1300$. Si se realiza una sencilla multiplicación entre el incremento de la TRM y el coeficiente estimado del modelo, se halla que el IPC debió incrementarse en $2,73 \%$. Esta cifra fue precisamente la diferencia aproximada entre la inflación observada en los últimos meses de 2016 y la observada en 2014. Por tanto, la TRM se constituye en una de las principales causantes de la inflación en Colombia, afirmándose de este modo la hipótesis estructuralista.
Una de las variables explicativas que llama la atención es la tasa de desempleo, debido al interés que surge por conocer la aplicación de la curva de Phillips al caso colombiano. Sin embargo, las estimaciones econométricas arrojaron una relación positiva entre el desempleo y la inflación, porque un incremento de $1 \%$ en la tasa de desempleo conduce a un incremento de $0,12 \%$ en la tasa de inflación, de manera que, inicialmente no se halla evidencia empírica de la relación negativa entre estas dos variables. Por esta razón, para validar la veracidad de la curva de Phillips hay que acudir a la curva de Phillips aumentada (Véase gráfico 6), donde se relaciona la tasa de desempleo con la diferencia entre la inflación esperada y la inflación observada; es decir que, se incluyen las expectativas.

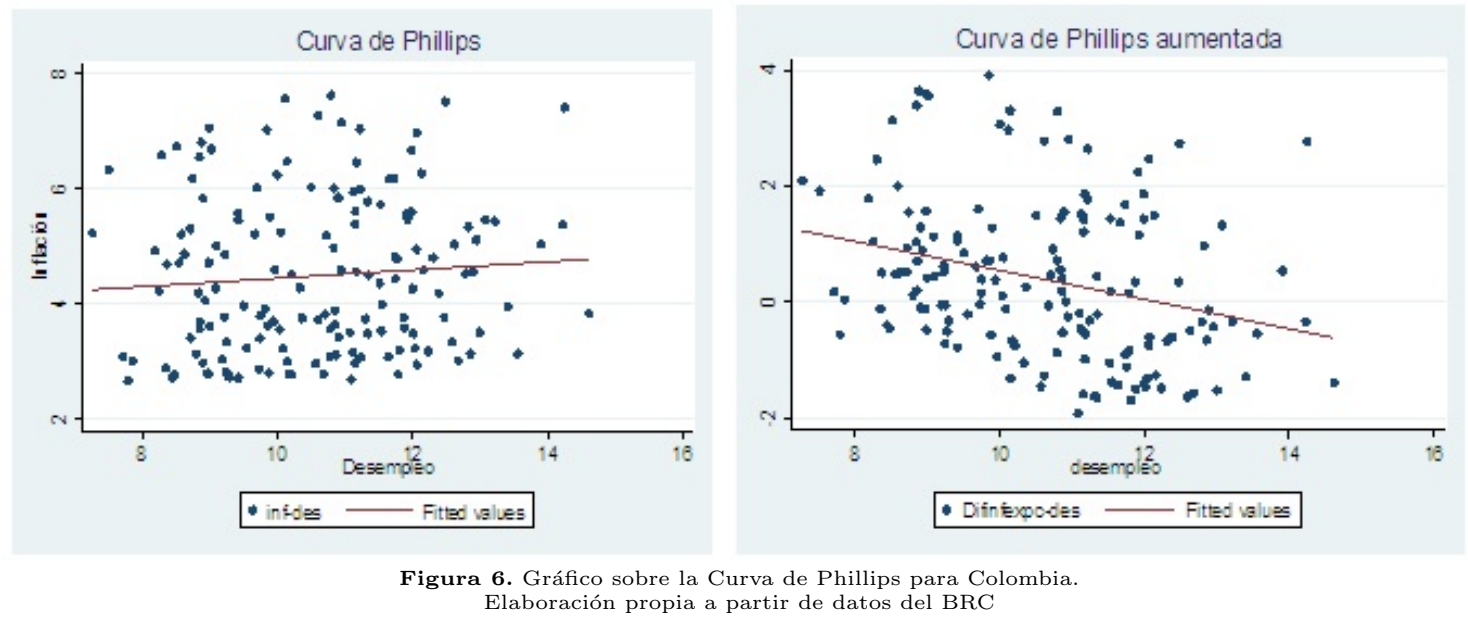

Los cálculos muestran que la tasa de desempleo tiene un efecto negativo sobre la variable que representa la diferencia entre la inflación esperada y la real. Por ello, si el desempleo aumenta $1 \%$ la desviación de la inflación se reduce en $-0,25 \%$, tal y como lo propone la teoría de la nueva curva de Phillips-Neokeynesiana; además, el parámetro estimado resulta ser estadísticamente significativo. De este modo, se halló evidencia empírica de la aplicabilidad de la curva de Phillips al caso colombiano, como también lo había hallado Galvis (2010).

Tabla 2. Estimación de la curva de Phillips aumentada

\begin{tabular}{|l|c|c|}
\hline Variables independiente & \multicolumn{2}{|c|}{ Variables dependiente } \\
\cline { 2 - 3 } & Inflación & Dif. Inflaexpect \\
\hline Desempleo & 0,0717 & $-0,2508^{* * *}$ \\
& $(0,017)$ & $(0,067)$ \\
\hline
\end{tabular}

ROJAS VELÁSQUEZ, L., \& CERQUERA LOSADA, ÓSCAR H. (2019)

Este es un artículo open Access bajo Licencia Creative Commons Atribución-No Comercial-Sin Derivadas 4.0 Internacional. 


\begin{tabular}{|l|c|c|}
\hline Intercepto & $3,7334^{* * *}$ & $3,0594^{* * *}$ \\
& $(0,753)$ & $(0,723)$ \\
\hline R-squared & 0,0067 & 0,0003 \\
\hline Prob $>$ F & 0,3097 & 0,0821 \\
\hline
\end{tabular}

Nota: ${ }^{* * *} \mathrm{p}$-value $<0,01 ;{ }^{* *} \mathrm{p}<0,05$. Entre paréntesis, el error estándar.

Cálculos propios a partir de datos del BRC

El crecimiento económico impacta positivamente el nivel general de precios, dado que un aumento de $1 \%$ en el PIB hace que la inflación aumente $0,13 \%$. Este resultado es de gran relevancia en términos económicos, porque una mayor actividad productiva incrementa la producción, el empleo, los salarios y por ende la disposición de dinero en manos del público, incrementando la demanda agregada y generando presiones sobre los precios; por tanto, una de las principales preocupaciones de las autoridades monetarios es mantener el equilibrio entre el aumento del PIB y el crecimiento de la inflación. En esta línea, la teoría keynesiana propone que el crecimiento económico no provoca en sí mismo la inflación, sino, que el aumento de los precios en general se origina a partir de la brecha absoluta entre el producto potencial y el producto observado. Así pues, en el presente ejercicio investigativo se estimó el PIB potencial a través del filtro de Hodrick-Prescott ${ }^{16}$; mientras que, la brecha del producto se estimó por medio de la siguiente ecuación:

$$
B P \%=\frac{\text { PIBobservdo }}{\text { PIBpotencial }}-1
$$

En este sentido, la brecha del producto tiene un efecto positivo sobre la inflación; de hecho, esta variable presenta el mayor coeficiente estimado en el modelo econométrico. Efectivamente, un aumento de un punto porcentual en la diferencia entre el PIB potencial y el PIB observado da lugar a un incremento de la inflación en $3,81 \%$. Incluso, como se muestra en el gráfico 7 , la diferencia entre el producto potencial y el observado aumentó notablemente en los periodos 2008-2009 y 2014-2016, precisamente cuando la tasa de cambio del IPC presentó sus dos picos más altos durante el periodo de análisis. Por su parte, en el periodo 2010-2013 la brecha inflacionaria absoluta fue muy baja, igual que el nivel general de precios. Según las estimaciones realizadas se pueden comprobar y afirmar los postulados keynesianos como explicación de la inflación en la economía colombiana.
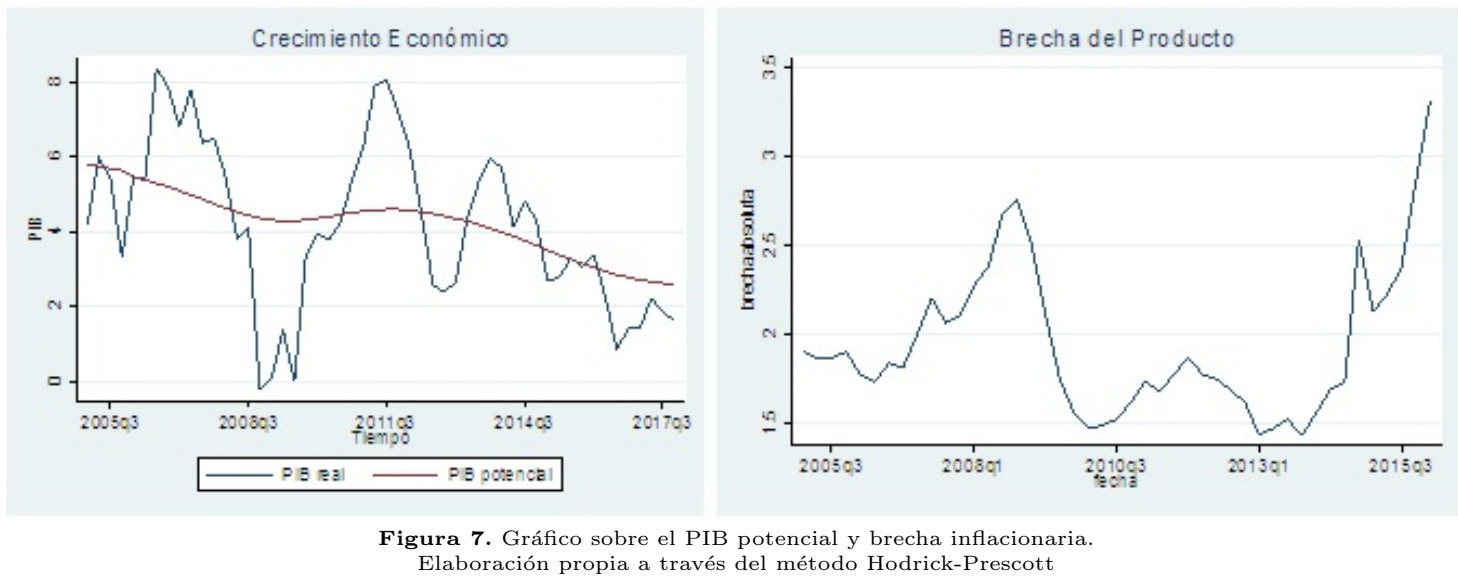

Por último, las externalidades originadas a al aumento del nivel general de precios. Más precisamente, la presencia del fenómeno del niño partir del comportamiento del clima están asociadas

ROJAS VELÁSQUEZ, L., \& CERQUERA LOSADA, ÓSCAR H. (2019)

Este es un artículo open Access bajo Licencia Creative Commons Atribución-No Comercial-Sin Derivadas 4.0 Internacional. 
impacta positivamente la inflación, al provocar un incremento de 0,25\%. Este resultado es lógico, dado que, en los periodos de mayor inflación se ha presentado este fenómeno climático. No sucede así con el fenómeno de la niña, como ya se mencionó previamente. Este hallazgo corrobora la evidencia empírica de Avella (2001) y Abril Salcedo, Melo Velandia y Parra Amado (2016).

\section{Conclusiones}

Las causas de la inflación en Colombia en su gran mayoría se asemejan a los diferentes postulados propuestos por las principales escuelas de pensamiento económico. En este sentido, se puede corroborar la tesis cuantitativa, porque los incrementos en la oferta monetaria han elevado el nivel de general de precios durante el periodo observado. Así mismo, se pudo identificar que la teoría keynesiana de la brecha inflacionaria se cumple en la economía colombiana. De igual manera, la inflación se incrementó en mayor proporción durante los periodos donde el PIB observado se alejó más de la capacidad potencial en valores absolutos; de hecho, el parámetro estimado para la variable que tipifica esta diferencia resultó ser el de mayor magnitud. También, se halló evidencia empírica para sustentar las teorías estructuralistas; en este caso la inflación surge como consecuencia de los desajustes del sistema productivo nacional; más precisamente, en Colombia parte de la inflación es importada; dado que, la TRM incide en el aumento del promedio general de los precios internos.

Por su parte, la curva de Phillips sencilla no se cumple para Colombia, pero la influencia del desempleo sobre la inflación sigue el comportamiento esperado cuando se estima la curva de Phillips aumentada, haciendo un ajuste a las expectativas de los agentes económicos.

Finalmente, las externalidades también han tenido su influencia sobre los precios en general; en efecto, en las épocas donde se presentaron oleadas de verano la inflación creció vertiginosamente. En este contexto, los resultados obtenidos evidencian una clara realidad para economía colombiana; por tanto, la política económica deberá estar orientada a superar las dificultades en el entorno monetario, los desfases cíclicos del producto y los desajustes estructurales que ponen la economía nacional en una posición de riesgo ante los choques externos.

\section{BIBLIOGRAFÍA}

Abril Salcedo, D. S., Melo Velandia , L. F., \& Parra Amado, D. (2016). Impactos de los fenómenos climáticos sobre el precio de los alimentos en Colombia. Ensayos sobre POLÍTICA ECONÓMICA Vol. 34 .

Avella, R. (2001). EFECTO DE LAS SEQUÍAS SOBRE LA INFLACIÓN EN COLOMBIA. Bogotá, Colombia : Subgerencia de Estudios Económicos del Banco de la República.

Banco de Mexico. (2012). Inflacion: cuasas, consecuencias y medicion. Mexico D.F.

BANREP. (2016). Informe sobre Inflacion. Bogotá: Equipo tecnico del Banco de la Republica de Colombia.

Bernanke, B., \& Frank, R. (2007). Macroeconomía. MxGraw-Hill.

Botero García, J., López Castaño, H., Posada , C. E. Ballesteros Ruíz , C., \& García Guzmán , J. (2015). ECONOMÍA COLOMBIANA: ANÁLISIS DE COYUNTURA. Medellín, Colombia: Escuela de Economía y Finanzas, Centro de Investigaciones Económicas y Financieras, Grupo de Investigación en Estudios en Economía y Empresa, Universidad EAFIT.

Cárdenas Pinzón, J. I., \& Vallejo Zamudio , L. (2012). Comportamiento de la inflación en Colombia 2002-2010 y régimen de metas de inflación. Apuntes del CENES, 33-54.

Consuegra Higgins, J. (2000). Teoría de la Inflación, el Interés y los salarios. Bogotá : Plaza \& Janés.

De Gregorio, J. (2000). Sobre los determinantes de la inflación y sus costos. Ensayos sobre politica economica del Banco de la Lepublica de Colombia: Revista ESPE, 27-61.

Dorbnusch, R., Fischer, S., \& Startz, R. (2008). Macroeconomía. McGraw-Hill.

Galvis , J. C. (2010). Estimación de la Curva de Phillips neokeynesiana para Colombia: 1990-2006. Lecturas de Economía No. 73, 11-47.

Jurado Estrada, J. A. (2005). Principales Teorías Económicas de la Inflación. Colombia. 
Landreth, H., \& Colander, D. (2006). Historia del pensamiento economico. España: McGraw-Hill.

Mankiw, G. (2006). Macroeconmía. España: Antoni Bosch Editor.

Marx, C. (1976). El Capital Tomo I. Mexico: Fondo de Cultura Económica.

Mendieta, P., \& Berbery, C. (2017). Entendiendo la Curva de Phillips del siglo XXI: estado de la cuestión. LAJED No. 28, 135-164.

Mimica, E. (2007). Los Efectos de la Inflación en la Evaluación de Proyectos de Inversión. Cambridge Resources International, Inc.South America.

Ochoa Díaz, H., \& Martínez Montealegre, Á. M. (2005). EL COMPORTAMIENTO DE LA INFLACIÓN EN COLOMBIA DURANTE EL PERÍODO 1955-2004. Estudios Gerenciales , 75-95.

Perez, C. (2011). Potencial de crecimiento y brecha de producto: variables clave para la politica económica. Bogotá: Investigaciones económicas del Banco de Bogotá.

Perossa, M., \& Waldman , P. (Noviembre de 2015). Causas y efectos de la inflación sobre la economía. Obtenido de ResearchGate: https://www.researchgate.net/publication/283422726

Causasyefectosdelainflacionsobrelaeconomia

Phillips, W. (1958). The Relation between Unemployment and the Rate of Change of Money Wage Rates in the United Kingdom, 1861-1957. Economica, New Series, Vol 25, No. 100, 283-299.

Pisani, F. (2013). La inflacion, el caballo de Troya del neoliberalismo. Ediciones "De un Tirón".

Ramírez Gallego, J. (2013). Estimación del producto potencial en Colombia: 1994-2012. Bogotá: Dirección de estudios Económicos del Departamento Nacional de Planeación.

Rodríguez, M., \& Zuloeta, J. (2008). Métodos alternativos para la estimación del PBI potencial 1950-2007. Centro de Investigación de la Universidad del Pacífico: Documento de discusión.

UNGRD. (2014). Plan Nacional de Contingencia ante el fenomeno del Niño 2014-2015. Bogotá: Unidad Nacional Para la Gestion del Riesgo de Desastres.

Wooldridge, J. (2010). Introducción a la econometría: un enfoque moderno. Michigan State University : CENAGE Lerning.

ROJAS VELÁSQUEZ, L., \& CERQUERA LOSADA, ÓSCAR H. (2019)

Este es un artículo open Access bajo Licencia Creative Commons Atribución-No Comercial-Sin Derivadas 4.0 Internacional. 